\title{
Super-twisting Control of Magnetic Levitation System
}

\author{
Rupak Rokade,* and Deepti Khimani, ** \\ ${ }^{1}$ Department of Instrumentation Engineering, V.E.S.Institute of Technology, Mumbai, India.
}

\begin{abstract}
This article presents the implementation results of second order sliding mode control (SOSM) for magnetic levitation system. In practical systems, especially when the actuators are electro-mechanical, the conventional (first order) sliding mode control can not be used effectively as it exhibits chattering, which is highly undesirable. Therefore, for such systems, sliding mode control of higher order can be a suitable choice as the reduce the chattering significantly. In this article the super-twisting control, which isa second order sliding mode control, is designed and implemented for the experimental setup of Maglev system, Model 730 developed by ECP systems.
\end{abstract}

\section{Introduction}

Reduction of the friction between moving surfaces is quite important aspect in electro-mechanical designs. Magnetic levitation principle provides an option of the noncontacting motion of moving objects, which substantially reduces the resistance to the motion. This increases the overall efficiency of the system.

Many industrial applications have been found in literature to have used a magnetic levitation successfully such as high-speed train suspension [1], magnetic bearings [2], launch assistance in space missions [3], superconductor rotor suspension of gyroscopes [4] and wind turbines $[5,6]$.

The control objective in such systems is to design the controller that maintains levitation of an object at a desired height. In past three decades various methods for the design of control for magentic levitation (maglev) systems have been reported in literature, for example, linear and nonlinear controller designs in [7, 8] via feedback linearization [9].

As the model uncertainties or the external disturbances may cause the system to underperform, it is desirable to design the robust controller that guarantees the desired performance in presence of disturbance. Numerous methods of robust controller design for Maglev system can be found in literature. For example, adaptive robust control design against parametric uncertainties [10], robust controller based on Lyapunov's direct method [3] or two DOF controller for magnetic levitation using quantitative feedback technique [11]. A sliding mode control (SMC) is one of popular robust control design methods for simplicity in control structure and insensitivity to a matched disturbances See $[12,13]$. Numerous articles on SMC design for Maglev system are found in literature. An experimental comparison of SMC and classical control has been found

\footnotetext{
*e-mail: rupak.rokade@ves.ac.in

**e-mail: deepti.khimani@ves.ac.in
}

in [14] and that of sliding mode disturbance compensation and $\mathrm{H}-\infty$ in [15]. In [16], SMC has been employed to provide high performance over a wide bandwidth in tracking of piezoelectric driven devices. Recently, experimental results on sliding mode control of maglev system has been discussed in [17]. The conventional (first order) sliding mode control exhibits chattering phenomenon due to it's discontinuous nature, which often is not suitable for practical applications. For a magnetic levitation system, SMC based on input-dependent surface to reduce the chattering has been discussed with simulation results in [18]. In [19], SMC has been designed with effective switching gain that is a function of distance between trajectory and the sliding surface. Super-twisting control (STC) is well known second order SMC, which reduces the chattering effect significantly [20-23]. This paper discusses the design and implementation of the STC on experimental setup Maglev Model 730 laboratory setup from educational control products [24]. The experimental results are compared with simulation results and are found satisfactory.

\section{System Description}

An experimental setup ECP Model 730 Maglev system [24] consists of two electromagnetic actuator coils, one mounted at the bottom that produces repulsive magnetic force to levitate the magnet and other actuator coil is mounted at the top, which produces the attractive magnetic force to that can be used to levitate the magnet. Each coil can be energized separately to levitate one or two magnets along a glass rod. Therefore, this system can either be configured as single-input single-output (SISO) or multiinput multi-output (MIMO) or multi-input single-output (SIMO) system. Fig.1 shows the representative diagram of experimental setup.

In this paper, we consider maglev system in SISO configuration and using lower coil to produces appropriate repulsive magnetic force that levitate the magnet at desired 


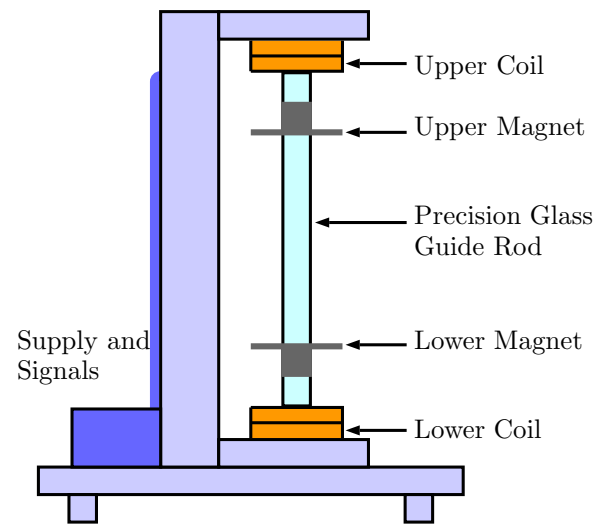

Figure 1. Maglev system, ECP-Model 730 representative diagram.

position while the upper coil is left unenergized. Fig.2 shows the free body diagram of ECP Model 730 Maglev system. The dynamical equation for the motion of the magnet with displacement $x(t)$ is given by,

$$
m \ddot{x}(t)+c \dot{x}(t)=F_{u}(t)-m g
$$

Where, $m$ is mass of magnet, $c$ is coefficient of viscous friction offered by glass rod during motion and $F_{u}(t)$ is repulsive force produced by the lower actuator (coil), which is nonlinear with respect to current $i$ through the coil and it is given by,

$$
F_{u}(t)=\frac{i(t)}{a(b+x(t))^{N}}
$$

Where $\mathrm{N}$, a and $\mathrm{b}$ are constants which can be determined via numerical approximation from the characteristics. Therefore, from (1) and (2), we get

$$
m \ddot{x}(t)+c \dot{x}(t)=\frac{i(t)}{a(b+x(t))^{N}}-m g
$$

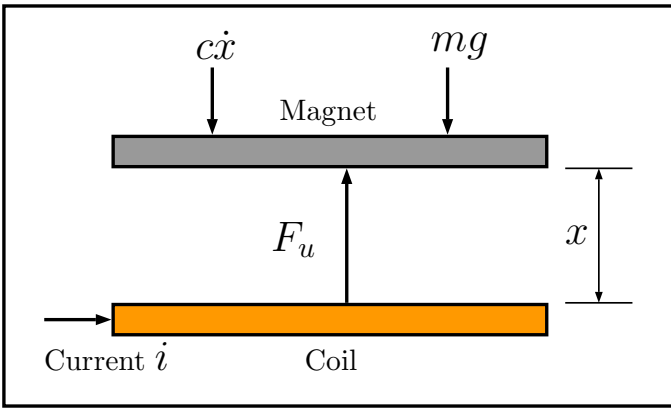

Figure 2. Free body diagram of ECP-Model 730 (SISO).

Define the state variables, $z_{1}:=x, z_{2}:=\dot{x}$ and control effort $u:=i$. So the system (3) can be represented in state variable form as,

$$
\begin{aligned}
\dot{z}_{1}(t) & =f_{1}(t)=z_{2}(t) \\
\dot{z}_{2}(t) & =f_{2}(t)=-\frac{c}{m} z_{2}(t)+\frac{u(t)}{m a\left(b+z_{1}(t)\right)^{N}}-g \\
y(t) & =z_{1}(t)
\end{aligned}
$$

\subsection{Linearization at operating point}

Let $u_{0}=i_{0}$ be the current passes through coil that levitate the magnet at desired height $x_{0}$. Therefore, at the operating point $\left(z_{1}, z_{2}, u\right)=\left(x_{0}, \dot{x}_{0}, u_{0}\right)=: O$. To linearize the system we perform Jacobian linearization at operating point. Follow from (4)-(5),

$$
\begin{aligned}
& J_{z}=\left[\begin{array}{ll}
\frac{\partial f_{1}}{\partial z_{1}} & \frac{\partial f_{1}}{\partial z_{2}} \\
\frac{\partial f_{2}}{\partial z_{1}} & \frac{\partial f_{2}}{\partial z_{2}}
\end{array}\right], \quad J_{u}=\left[\begin{array}{c}
\frac{\partial f_{1}}{\partial u} \\
\frac{\partial f_{2}}{\partial u}
\end{array}\right] \\
& J_{z}=\left[\begin{array}{cc}
0 & 1 \\
\frac{-N u(t)}{a\left(b+z_{1}(t)\right)^{N+1}} & -\frac{c}{m}
\end{array}\right], \quad J_{u}=\left[\begin{array}{c}
0 \\
\frac{1}{a(b+x(t))^{N}}
\end{array}\right]
\end{aligned}
$$

Then the linearized model at operating point is given by,

$$
\left[\begin{array}{c}
\dot{z}_{1}(t) \\
\dot{z}_{2}(t)
\end{array}\right]=\left.J_{z}\right|_{O}\left[\begin{array}{c}
z_{1}(t) \\
z_{2}(t)
\end{array}\right]+\left.J_{u}\right|_{O} u(t)
$$

This gives,

$$
\begin{aligned}
{\left[\begin{array}{c}
\dot{z}_{1}(t) \\
\dot{z}_{2}(t)
\end{array}\right] } & =\left[\begin{array}{ll}
A_{11} & A_{12} \\
A_{21} & A_{22}
\end{array}\right]\left[\begin{array}{c}
z_{1}(t) \\
z_{2}(t)
\end{array}\right]+\left[\begin{array}{c}
0 \\
B_{2}
\end{array}\right] u(t) \\
y & =z_{1}(t)
\end{aligned}
$$

Where,

$$
\begin{aligned}
& A_{11}=0, A_{12}=1, A_{21}=\frac{-N u_{0}}{m a\left(b+x_{0}\right)^{N+1}} \\
& A_{22}=-\frac{c}{m} \text { and } B_{2}=\frac{1}{m a\left(b+x_{0}\right)^{N}}
\end{aligned}
$$

Remark 2.1 In ideal condition, $c=0$ i.e there is no air resistance and no friction between glass rod and magnet, the system can be represented as,

$$
\begin{aligned}
{\left[\begin{array}{c}
\dot{z}_{1}(t) \\
\dot{z}_{2}(t)
\end{array}\right] } & =\left[\begin{array}{cc}
0 & 1 \\
A_{21} & 0
\end{array}\right]\left[\begin{array}{l}
z_{1}(t) \\
z_{2}(t)
\end{array}\right]+\left[\begin{array}{c}
0 \\
B_{2}
\end{array}\right] u(t) \\
y(t) & =z_{1}(t)
\end{aligned}
$$

Note that, the response of the system (11) is oscillatory about the operating point because the poles are pure imaginary at $\pm j \sqrt{\left|A_{21}\right|}$. However, practically, there exist a finite amount of resistance that can be modeled as viscous friction, though very small. This results in underdamped transient response.

\section{System identification}

For the linear model (11), the mass of magnet and gravitational force are known. However, parameters $a, b$ and $N$ are required to compute $A_{21}$ and $B_{2}$. For the experimental setup, the values recommended by manufacturer are $b=6.2$ and $3<N<4.5$. With these values, the constant $a$ can be computed using the curve-fitting of actuator (2) response for input $u$ and the magnet position $x$. As the steady-state height of the magnet represents an equilibrium, i.e. $F_{u}=m g$, therefore from (2) we get,

$$
a=\frac{u}{m g\left(b+z_{1}\right)^{N}}
$$


Table 1. Parameters of Maglev system

\begin{tabular}{|c|c|c|}
\hline Parameter & Quantity & Remark \\
\hline$m$ & $0.12 \mathrm{Kg}$ & mass of magnet \\
\hline$N$ & 4 & selected \\
\hline$b$ & 6.2 & provided by manufacturer \\
\hline$g$ & 9.81 & $\mathrm{~m} / \mathrm{s}^{2}$ \\
\hline
\end{tabular}

The value of constant $a$ for the pair $(x, u)$ can be computed from (12) and data in Table 1. The mean value is found to be $a=0.99$ after curve fitting. Based on these parameters, the linear model at $x_{0}=2 \mathrm{~cm}$ and $u_{0}=5000$ count $\mathrm{s}$ can be identified as,

$$
\begin{aligned}
& \dot{x}(t)=\left[\begin{array}{cc}
0 & 1 \\
-313 & -6.5
\end{array}\right] x(t)+\left[\begin{array}{c}
0 \\
5000
\end{array}\right] u(t) \\
& y(t)=z_{1}(t)
\end{aligned}
$$

Fig. 3 shows open loop responses of the system, obtained practically as well as via simulation.

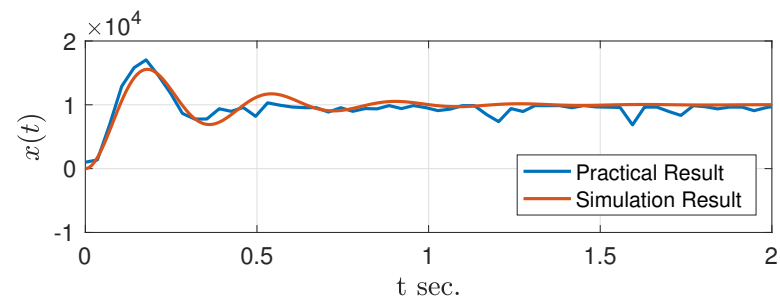

Figure 3. Open loop response of the Levitation $y(t)$ of magnet.

\section{Super-twisting control}

Consider a controllable system,

$$
\begin{aligned}
& \dot{\mathbf{z}}_{1}(t)=A_{11} \mathbf{z}_{1}(t)+A_{12} z_{2}(t) \\
& \dot{z}_{2}(t)=A_{21} \mathbf{z}_{1}(t)+A_{22} z_{2}(t)+B_{2} u(t)+d(\mathbf{z}, t)
\end{aligned}
$$

where, $\mathbf{z}_{1} \in \mathbf{R}^{n-1}, z_{2} \in \mathbf{R}, z=\left[\mathbf{z}_{1}^{\top} z_{2}\right]^{\top}$ represent states of the system, $u \in \mathbf{R}$ and $d \in \mathbf{R}$ represent input and disturbance, respectively. Assume that, the system is controllable and observable. Also, assume that the disturbance and its rate of change with respect to time are bounded by, $|d(\mathbf{z}, t)| \leq \gamma$ and $\phi(z, t):=|\dot{d}(\mathbf{z}, t)| \leq \gamma_{1}$, respectively.

\subsection{Sliding Function and Sliding Mode Control}

Let there exist $c_{1} \in \mathbf{R}^{1 \times(n-1)}$ such that $\left(A_{11}-A_{12} c_{1}\right)$ is Hurwitz. Such a matrix $F$ exists as the $\left(A_{11}, A_{12}\right)$ pair inherits the controllability property.

Let the sliding set be,

$$
\mathbf{S}=\left\{\mathbf{z} \in \mathbf{R} \mid s(t):=c_{1} \mathbf{z}_{1}(t)+z_{2}(t)=0\right\}
$$

Clearly, when the state trajectory is held on the surface $s(t)=0$, we get $z_{2}=-c_{1} \mathbf{z}_{1}$. Therefore, from (14a) we can write governing equation for sliding motion of $\mathbf{z}_{1}$ (and subsequently, $\mathbf{z}_{2}=-c_{1} \mathbf{z}_{1}$ ) trajectory as

$$
\dot{\mathbf{z}}_{1}(t)=\left(A_{11}-A_{12} c_{1}\right) \mathbf{z}_{1}(t)
$$

As $\left(A_{11}-A_{12} c_{1}\right)$ is Hurwitz by design, thus when trajectory of the system is held on $s(t)=0,\left\|\mathbf{z}_{1}\right\| \rightarrow 0$ as $t \rightarrow \infty$ and $\left\|z_{2}\right\| \rightarrow 0$ as $\left\|\mathbf{z}_{1}\right\| \rightarrow 0$.

Therefore, it is essential to design the control such that the trajectory $\left(\mathbf{z}_{1}, z_{2}\right)$ reaches the sliding surface $s(t)$ in finite time and remain on that thereafter. For such systems, the conventional sliding mode control that enforces the system trajectory on the surface in finite time and restrict them on it can be given by,

$$
\begin{aligned}
u(t)=- & B_{2}^{-1}\left(c_{1}\left[A_{11} \mathbf{z}_{1}(t)+A_{12} z_{2}(t)\right]\right. \\
& \left.+A_{21} \mathbf{z}_{1}(t)+A_{22} z_{2}(t)+Q \operatorname{sgn}(s)\right), Q>\gamma
\end{aligned}
$$

The conventional sliding mode control (17) ensures $s(t)=0$ in a finite time. However, the term $Q \operatorname{sgn}(s)$ makes the control $u$ discontinuous and may exhibit the chattering. This problem can be overcome by using second order sliding mode, in which the time derivative of the control is considered to be the new virtual control that ensures $\dot{s}(t)=0$ in finite time as well.

From (15), we can write,

$$
\begin{aligned}
\dot{s}(t)= & c_{1} \dot{\mathbf{z}}_{1}(t)+\dot{z}_{2}(t) \\
= & c_{1}\left[A_{11} \mathbf{z}_{1}(t)+A_{12} z_{2}(t)\right]+A_{21} \mathbf{z}_{1}(t) \\
& +A_{22} z_{2}(t)+B_{2} u(t)+d(\mathbf{z}, t)
\end{aligned}
$$

Define,

$$
f(t):=c_{1}\left[A_{11} \mathbf{z}_{1}(t)+A_{12} z_{2}(t)\right]+A_{21} \mathbf{z}_{1}(t)+A_{22} z_{2}(t)
$$

Therefore, from (18) we can write,

$$
\dot{s}(t)=f(t)+B_{2} u(t)+d(\mathbf{z}, t)
$$

Clearly, objective is to design the control such that, $s(t)=$ $\dot{s}(t)=0$ for all $t \geq t_{r}, t_{r} \in \mathbf{R}^{+}$.

\subsection{Super-twisting Control}

The first order (conventional) SMC as in (17) is discontinuous in nature. Practically such discontinuous control cannot be applied at infinite frequency, this leads to the chattering that may excite unmodelled dynamics and as a result system may under perform or may result into the actuator failure. In order to obtain the sliding motion as in (16) along with robustness properties, a super-twisting control can be used, which circumvents the chattering issue. Typically, the super-twisting structure is given by,

$$
\begin{aligned}
& \dot{s}(t)=-k_{1}|s|^{\frac{1}{2}} \operatorname{sgn}(s)+\xi(t) \\
& \dot{\xi}(t)=-k_{2} \operatorname{sgn}(s)+\phi(t)
\end{aligned}
$$

Then $s(t)$ and $\dot{s}(t)$ converge to zero in finite time if $k_{1}$ and $k_{2}$ are chosen as, $k_{2}>\gamma_{1}$ and $k_{1}>1.8 \sqrt{\gamma_{1}+k_{2}}$, see [23] for proof. 
Clearly, if we design a control,

$$
u(t)=-B_{2}^{-1}\left(f(t)+k_{1}|s|^{\frac{1}{2}} \operatorname{sgn}(s)+k_{2} \int_{0}^{t} \operatorname{sgn}(s) d \tau\right)
$$

Then (19) can be written as,

$$
\dot{s}(t)=-k_{1}|s|^{\frac{1}{2}} \operatorname{sgn}(s)-k_{2} \int_{0}^{t} \operatorname{sgn}(s) d \tau+d(\mathbf{z}, t)
$$

Define, $\xi:=-k_{2} \int_{0}^{t} \operatorname{sgn}(s) d \tau+d(\mathbf{z}, t)$ and $\phi(t):=\dot{d}(\mathbf{z}, t)$ Therefore (22) can be written as,

$$
\begin{aligned}
& \dot{s}(t)=-k_{1}|s|^{\frac{1}{2}} \operatorname{sgn}(s)+\xi(t) \\
& \dot{\xi}(t)=-k_{2} \operatorname{sgn}(s)+\phi(t)
\end{aligned}
$$

Thus, $s(t)=\dot{s}(t)=0$ for all $t>t_{r}$ for some $t_{r} \in \mathbf{R}^{+}$if we choose $k_{2}>\gamma_{1}$ and $k_{1}>1.8 \sqrt{\gamma_{1}+k_{2}}$.

\section{Sliding mode and super-twisting control for maglev system}

This section demonstrates the simulation and practical responses of magnetic levitation system with sliding mode control and super-twisting control. Both the controllers are designed for the identified system as in (13).

Comparing (9) and (13), we get,

$$
\begin{aligned}
& A_{11}=0, A_{12}=1, A_{21}=-313 \\
& A_{22}=-6.5 \text { and } B_{2}=5000
\end{aligned}
$$

To track the reference input $r$, we transform the system to new co-ordinates that represent error between system states and the desired states. Define $e_{1}:=z_{1}-z_{1 d}=z_{1}-r$, $e_{2}:=z_{2}-z_{2 d}$, and $\Delta u:=u-u_{d}$. So the system (9) can be represented as,

$$
\begin{aligned}
& \dot{e}_{1}(t)=A_{11} e_{1}(t)+A_{12} e_{2}(t) \\
& \dot{e}_{2}(t)=A_{21} e_{1}(t)+A_{22} e_{2}+B_{2} \Delta u(t)
\end{aligned}
$$

\subsection{Sliding function}

From (15), we can design sliding function as,

$$
s(t)=c_{1} e_{1}(t)+e_{2}(t)=0
$$

such that the sliding motion governed by

$$
\dot{z}_{1}(t)=\left(A_{11}-A_{12} c_{1}\right) z_{1}(t)
$$

will exhibit the desired characteristic. We choose, $c_{1}=10$, therefore for the maglev system surface is given by

$$
s(t)=10 e_{1}(t)+e_{2}(t)=0
$$

\subsection{Sliding mode control}

Follow from (17), we can design the sliding mode control for the system (25)-(26) that initiates the sliding motion in finite time,

$$
\begin{aligned}
u(t)=- & B_{2}^{-1}\left(c_{1}\left[A_{11} e_{1}(t)+A_{12} e_{2}(t)\right]\right. \\
& \left.+A_{21} e_{1}(t)+A_{22} e_{2}(t)+Q \operatorname{sgn}(s)\right)
\end{aligned}
$$

Where $Q$ is chosen greater than bound of the absolute value of disturbance and $k$ is chosen to increase the speed of response during reaching phase. To check the robustness property of the sliding mode control, disturbance $d(t)=2 \sin 20 t$ is introduced. Therefore, we select $Q=$ $5>2$ and $k=40$ to speed up the response in the reaching phase. Thus, the sliding mode control is designed as,

$$
\begin{aligned}
u(t)= & 0.626 e_{1}(t)-0.0007 e_{2}(t)-0.008 s(t) \\
& -0.001 \operatorname{sgn}(s)
\end{aligned}
$$

that initiates the sliding motion

$$
\dot{z}_{1}(t)=-10 z_{1}(t)
$$

in finite time. Clearly, $\left\|e_{1}\right\| \rightarrow 0$ as $t \rightarrow \infty$ and as $e_{2}(t)=$ $-10 e_{1}$ therefore $\left\|e_{2}\right\| \rightarrow 0$ as $t \rightarrow \infty$. Thus, $y=z_{1}$ tracks $r$ asymptotically.

Fig.4 shows the comparison of simulation response with the response of magnet levitation performed on experimental setup ECP Maglev 730. Fig.4 shows the control efforts (31) and evolution of sliding function (29) for both simulation and practical performance.

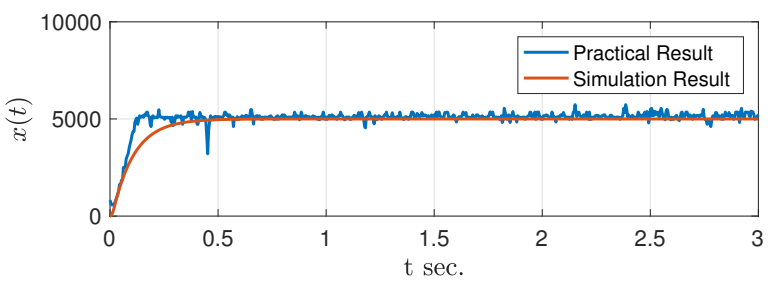

Figure 4. Levitation $x(t)$ of magnet with sliding mode control (31).

\subsection{Super-twisting control}

Form Fig.5 (a), it is quite evident that the applied control exhibits the chattering, therefore we design super-twisting control, which is second order continuous control as discussed in section 4.2. Follow from (21), we can write the super-twisting control for a system (25)-(25) described in error variables as,

$$
u(t)=-B_{2}^{-1}\left(f(t)+k_{1}|s|^{\frac{1}{2}} \operatorname{sgn}(s)+k_{2} \int_{0}^{t} \operatorname{sgn}(s) d \tau\right)
$$

where,

$$
f(t)=c_{1}\left[A_{11} e_{1}(t)+A_{12} e_{2}(t)\right]+A_{21} e_{1}(t)+A_{22} e_{2}(t)
$$



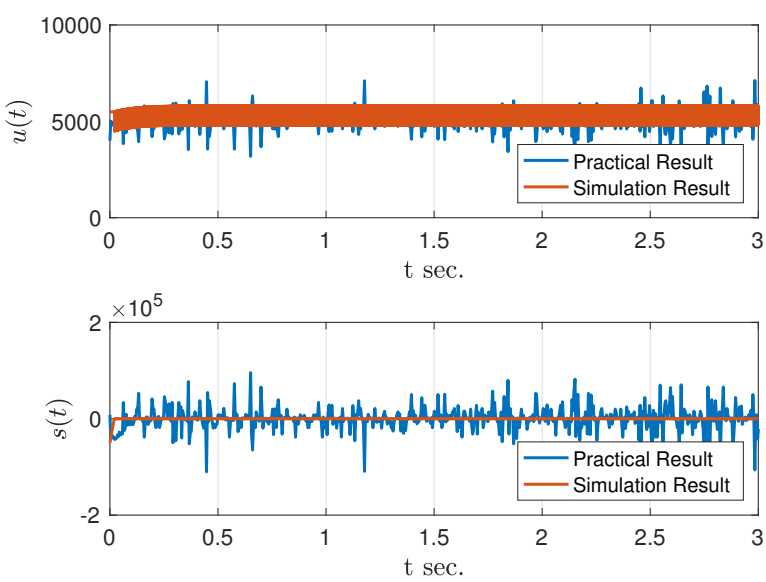

Figure 5. (a) Control input (31) to the system. (b) Evolution of sliding surface (29).

Thus, for experimental setup ECP Maglev 730 with disturbance $d(t)=2 \sin 20 t$, we design a control with $k_{1}=$ 16000 and $k_{2}=10$ as,

$$
\begin{aligned}
u(t)= & 0.626 e_{1}(t)-0.0007 e_{2}(t)-3.2|s|^{\frac{1}{2}} \operatorname{sgn}(s) \\
& +0.002 \int_{0}^{t} \operatorname{sgn}(s) d \tau
\end{aligned}
$$

that provides the super-twisting control in terms of counts as,

$$
\begin{aligned}
& \dot{s}(t)=-16000|s|^{\frac{1}{2}} \operatorname{sgn}(s)+\xi(t) \\
& \dot{\xi}(t)=-10 \operatorname{sgn}(s)+\phi(t)
\end{aligned}
$$

Note that, $\max |\phi(t)|=\max |\dot{d}|=2$, therefore $k_{2}>2.2$ is satisfied and $k_{1}>1.8 \sqrt{2.2+10}$ is also satisfied. As $s$ and $\dot{s}$ both converge to 0 in finite time, sliding motion (32) is initiated. This implies, $\left\|e_{1}\right\| \rightarrow 0$ as $t \rightarrow \infty$ and as $e_{2}(t)=-10 e_{1}$ therefore $\left\|e_{2}\right\| \rightarrow 0$ as $t \rightarrow \infty$. Thus, $y=z_{1}$ tracks $r$ asymptotically. Fig.6 shows the comparison of simulation response and the response of magnet levitation performed on experimental setup ECP Maglev 730 with super-twisting control (34). Fig.7 shows the control efforts (34) and evolution of sliding function (29) for both simulation and practical performance. It can be seen in Fig.7(a) that control effort (34) does not exhibit chattering.

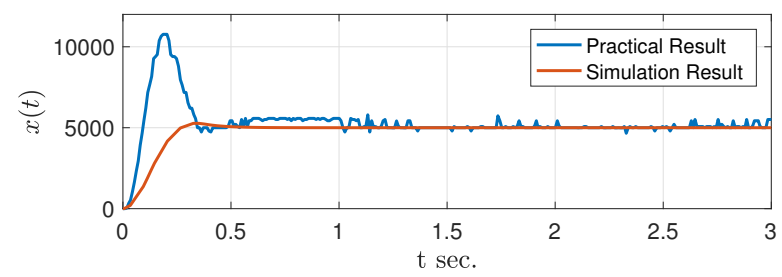

Figure 6. Levitation $x(t)$ of magnet with sliding mode control (34).
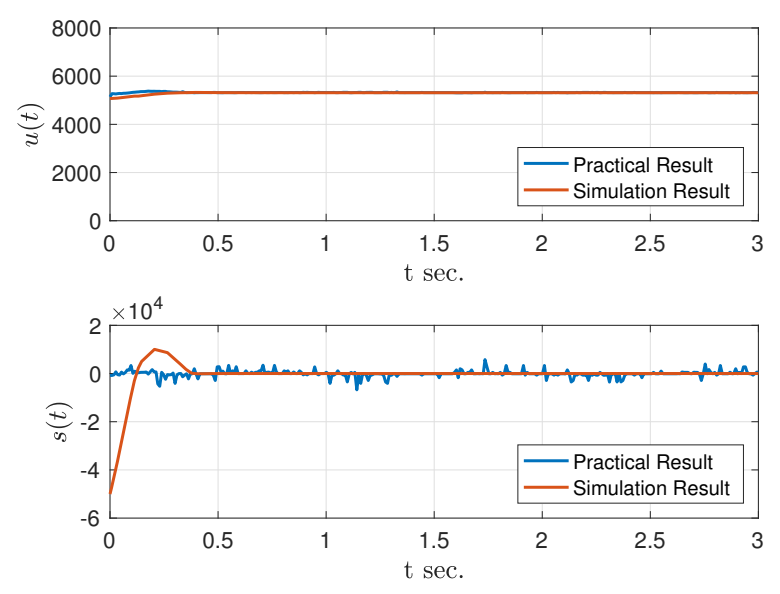

Figure 7. (a) Control input (34) to the system. (b) Evolution of sliding surface (29).

\section{Conclusion}

In this paper, we have designed and implemented the sliding mode (SMC) and super-twisting control (STC) for the Maglev Model 730 experimental setup manufactured by educational control products, which is an open loop underdamped system. The experimental results with SMC and STC are compared with simulation results and found satisfactory in presence of external disturbance.

It can be verified from the simulation as well as practical results that STC is continuous control and does not exhibit chattering and the manget levitation response does not hinder in presence of applied disturbance.

\section{References}

[1] B.Z. Kaplan, D. Regev, IEEE Transactions on Magnetics 12, 556 (1976)

[2] M. Dussaux, Status of the industrial applications of the active magnetic bearings technology, in ASME 1990 International Gas Turbine and Aeroengine Congress and Exposition (American Society of Mechanical Engineers, 1990), pp. V005T14A016V005T14A016

[3] J. Kaloust, C. Ham, J. Siehling, E. Jongekryg, Q. Han, IEE Proceedings-Control Theory and Applications 151, 460 (2004)

[4] W.J. Bencze, Y.M. Xiao, D.N. Hipkins, G.F. Franklin, B.W. Parkinson, Gyroscope spin axis direction control for the Gravity Probe $B$ satellite, in Decision and Control, 1996., Proceedings of the 35th IEEE Conference on (IEEE, 1996), Vol. 1, pp. 480485

[5] S.Y. Hu, Magnetic levitation weight reduction structure for a vertical wind turbine generator (2008), uS Patent 7,462,950

[6] J. Kumbernuss, C. Jian, J. Wang, H. Yang, W. Fu, Applied Energy 90, 148 (2012) 
[7] W. Barie, J. Chiasson, International Journal of systems science 27, 1153 (1996)

[8] A. El Hajjaji, M. Ouladsine, IEEE Transactions on industrial Electronics 48, 831 (2001)

[9] A. Isidori, Nonlinear control systems (Springer Science \& Business Media, 2013)

[10] Z.J. Yang, M. Tateishi, Automatica 37, 1125 (2001)

[11] P. Nataraj, M.D. Patil, Robust control design for nonlinear magnetic levitation system using quantitative feedback theory (QFT), in India Conference, 2008. INDICON 2008. Аnnual IEEE (IEEE, 2008), Vol. 2, pp. $365-370$

[12] C. Edwards, S.K. Spurgeon, Sliding Mode Control: Theory and Applications (Taylor \& Francis Ltd., 1998)

[13] Y. Shtessel, C. Edwards, L. Fridman, A. Levant, Sliding mode control and observation (Springer, 2014)

[14] D. Cho, Y. Kato, D. Spilman, IEEE control systems 13, 42 (1993)

[15] J.C. Shen, Asian Journal of Control 4, 333 (2002)

[16] H.M. Gutierrez, P.I. Ro, IEEE Transactions on Industrial Electronics 45, 921 (1998)
[17] D. Khimani, R. Rokade, Implementation of sliding mode control on magnetic levitation system, in Advances in Computing, Communication and Control (ICAC3), 2017 International Conference on (IEEE, 2017), pp. 1-5

[18] N. Al-Muthairi, M. Zribi, Mathematical Problems in Engineering 2004, 93 (2004)

[19] J. Phuah, J. Lu, T. Yahagi, IEEJ Transactions on Electronics, Information and Systems 125, 600 (2005)

[20] A. Levant, Automatica 34, 379 (1998)

[21] A. Polyakov, A. Poznyak, IEEE Transactions on Automatic Control 54, 1951 (2009)

[22] J.A. Moreno, M. Osorio, IEEE Transactions on Automatic Control 57, 1035 (2012)

[23] A.K. Behera, A. Chalanga, B. Bandyopadhyay, Automatica 87, 437 (2018)

[24] ECP, Operational Manual for Magnetic Levitation system-Model 730 (ECP Systems, 2015), http:// www.ecpsystems.com/controls $\backslash$ maglevit.htm 